\title{
Discrete quasi-gradient features weighting algorithm
}

\author{
Norbert Jankowski \\ Department of Informatics, Nicholas Copernicus University \\ ul. Grudziadzka 5, 87-100 Toruń, Poland, http://www.phys.uni.torun.pl/ ${ }^{\sim}$ norbert
}

\begin{abstract}
A new method of feature weighting, useful also for feature extraction has been described. It is quite efficient and gives quite accurate results. Weighting algorithm may be used with any kind of learning algorithm. The weighting algorithm with k-nearest neighbors model was used to estimate the best feature base for a given distance measure. Results obtained with this algorithm clearly show its superior performance in several benchmark tests.
\end{abstract}

\section{Introduction}

It is well known [12] that initial feature set of data sets/databases used for classification or approximation is not the optimal information source and the feature analysis may be very helpful in further processing of data. Several methods which use different strategy of feature extraction and weighting were already presented in books and articles 3415. Some of them measure amount of information belonging to a given attribute (or a subset 1 ) using information theory or statistics. Other methods use learning models to observe accuracy changes what help to estimate weights in the next phase of feature weighting (or extraction) process 674. Such type of feature weighting may be used with several learning models types. Algorithm presented in this article belongs to the second type of feature weighting/extracting algorithms, and will be presented in conjunction with k-nearest neighbors model [8], although algorithm itself may be used with any learning model.

\section{Feature weighting algorithm}

General idea of a Discrete quasi-gradient algorithm is based on looking for optimal vector of weight changes in given state of weighting procedure. The process is repeated as long as any improvement of accuracy may be done. In parallel some control parameters change to stimulate quasi-gradient directions of weights changes. Another goal of this algorithm was to create stable feature weighting algorithm. It means that new (weighted) set of features should never significantly decrease accuracy of the final model. Of course it is impossible to expect any progres if original feature set is optimal. Next,

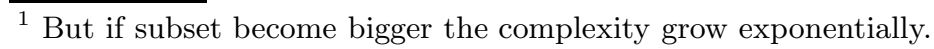


repeating the whole procedure of weighting a few distinguishable solutions may be found. This is not an error, it is nature of some databases. Such information may be important in propagation of this information (separate weighted sets of features) to other models and it may help to obtain different final models.

The main loop of the algorithm consists of cross-validation (CV) as a learning technic. This means that a whole data set is divided in $n$ equal (if possible) parts (commonly called folds) $\mathcal{S}_{i}, i=1, \ldots, n$. In each CViteration procedure FindWeights is called. FindWeights use two sets $\hat{\mathcal{S}}_{i}$ $\left(\hat{\mathcal{S}}_{i}=\bigcup_{k=1, \ldots, n, k \neq i} \mathcal{S}_{k}\right)$ as a learning set, and $\mathcal{S}_{i}$ as a validation set. As a result procedure FindWeights returns vector of feature weights $\mathbf{w}_{i}$. Lets define:

$$
\mathbf{w}_{i}=\text { FindWeights }\left(\hat{\mathcal{S}}_{i}, \mathcal{S}_{i}\right)
$$

Each weight of $\mathbf{w}_{i}$ is in $[0,1]$ interval. Details of procedure FindWeights will be presented later. Vectors $\mathbf{w}_{i}$ consist of weights for each feature $k=1, \ldots, d$, where $d$ is the dimension of data set $\mathcal{S}$. Now the sum of weights vectors is calculated and the final weights vector is obtained through normalization:

$$
\tilde{\mathbf{w}}=\mathbf{w} / \max _{k=1, \ldots, d} w_{k} \quad \mathbf{w}=\sum_{i=1}^{n} \tilde{\mathbf{w}}_{i}
$$

where $\mathbf{w}=\left[w_{1}, \ldots, w_{d}\right]$. The above part of CV learning is really simple: if all $\mathrm{CV}$ iterations are completed, the final model is estimated as a consequence of intermediate models. Now the FindWeights as the heart of whole algorithm will be described.

Procedure FindWeights. First, the initial values are assigned to weights: $w_{k}=1, \quad k=1, \ldots, d$. In this case the algorithm starts from full feature set. It may be useful sometimes to start from $w_{k}=0$, what mean that algorithm starts from an empty feature set and tries to add feature by feature - this strategy may be more efficient, computationally, if working with highly dimensional data sets. The main loop (one of three) is repeated as long as any progress in the maximization of accuracy on validation set may be done. The contents of that loop is called main phase. The goal of the main phase is to observe changes, determining which features may help to improve the accuracy by changing current weights by $\Delta$, and next to apply positive/progresive changes and simultaneously coordinating the change of $\Delta$. In the inner loop for each feature two quantities $v_{i}^{+}$and $v_{i}^{-}$are computed:

$$
v_{i}^{+}=\operatorname{validate}\left(\mathbf{w}^{+}, i\right) \quad v_{i}^{-}=\operatorname{validate}\left(\mathbf{w}^{-}, i\right)
$$

where $\mathbf{w}^{ \pm}=\left[w_{1}, \ldots, w_{i-1}, w_{i} \pm \Delta, w_{i+1}, \ldots, w_{d}\right]$. validate $(\mathbf{w})$ is a function which compute the accuracy on validation set $\mathcal{S}$ (see eq. [1) for the learning model $\mathcal{M}$ which is trained on a data set $\hat{\mathcal{S}}$. To obtain results presented in section 3 the k-nearest neighbor model was used as $\mathcal{M}$.

Vectors $\mathbf{v}^{+}$and $\mathbf{v}^{-}$contain information about weight changes by step $\Delta$ that may help to improve results. If any $v_{i}^{ \pm}$indicate that given change may 
help the new set of weights is defined according to:

$$
\begin{array}{lll}
w_{i}^{\prime}=w_{i}-\theta \Delta & \text { if } \quad v_{i}^{-}>v \\
w_{i}^{\prime}=w_{i}+\theta \Delta & \text { if } \quad v_{i}^{+}>v \wedge v_{i}^{-}<=v
\end{array}
$$

where $\theta$ defines the speed (I like $\theta=1$ - really fast!), and $v=$ validate $(\mathbf{w})$.

The above equation says that all features which may help if they are changed, are indeed changed. If for new weights $v_{\text {new }}=\operatorname{validate}\left(\mathbf{w}^{\prime}\right)$ is smaller than $v=$ validate $(\mathbf{w})$ the procedure goes back to weights $\mathbf{w}$, else $\mathbf{w}^{\prime}$ become the new weight vector.

As long as the current parameter $\Delta$ leads to a better accuracy, weight changes are made and the inner loop is continued. Usually the starting $\Delta$ is equal to 1 . The step $\Delta$ is decreased if no progress in maximization of accuracy on validation set is done: $\Delta=\Delta / 2$. After that if $\Delta$ is still bigger (or equal to) than $\Delta_{\min }$, the second loop is repeated again. If $\Delta$ is smaller than $\Delta_{\text {min }}$ (for example 0.01) loop is broken and weights are normalized:

$$
\tilde{\mathbf{w}}=\mathbf{w} / \max _{k=1, \ldots, d} w_{k}
$$

The main phase loop is repeated (with initial $\Delta=1$ ) as long as the weighting algorithm gives with better accuracy on a validation test. Since the main phase loop is repeated the algorithm is able to jump out from local minima keeping the best solution found so far. Without that loop the performance of the algorithm was average or even worst.

Outline of this algorithm may be found at my WWW page.

\section{Results}

The performance on several databases was checked to test the stability of the algorithm and to test whether feature weighting helps or may destroy the generalization ability. All test were performed on databases from Machine Learning Database Repository at the UCI [9] using 10-fold CV test, except that data sets which have original testing sets. All results were averaged from 10 runs. Standardization of most database was performed before the learning phase. Each table with results have four parts. First presents results which were obtained with feature weighting algorithm for kNN, second without feature weighting, and third show default accuracy - percentage of most majority class. Fourth shows results obtained by other models. This part contains only the best models for a given databas 23. Note that k-weight [10] and GIBL [7] are algorithms which estimate feature influence. It is common that some models have really good accuracy for one benchmark giving much worst results for other data sets. In tables with results the notation is used:

\footnotetext{
${ }^{2}$ If you want to browse more results see WWW pages of my LAB: http://www.phys.uni.torun.pl/kmk/projects/datasets.html.
} 
$\mathrm{kNN}[\mathrm{CVx}]$ [M] . k defines the number of neighbors. $C V x$ if exists means that weighting algorithm was used, and $x$ defines the number of folds in $\mathrm{CV}$. If $M$ occurs non-Euclidian metric was used, such as the Manhattan, Minkovsky or Canberra 3 . For some data set significant improvement of accuracy on test sets is observed, and for others data sets the accuracy is not (essentially) changed while for others the classification model decreases its accuracy.

Thyroid disease is one of the best known benchmark data sets, as well one really nontrivial for classification. The training set has 3772 cases, and testing set has 3428 cases. There are 3 classes, and the most frequent has $92.71 \%$ of all cases. Number of attributes is 21 (15 binary, 6 continuous). The accuracy on test set of plain $1 \mathrm{NN}$ ( $\mathrm{kNN}$ with 1 neighbor) is really poor, just $93.14 \%$ (see tab. 1). For $3 \mathrm{NN}$ with Manhattan distance the accuracy is $94.4 \%$, but using weighted features $98.36 \%$. The best results were obtained for $3 \mathrm{NN}$ with the Canberra metric $-98.56 \%$. This very good results were obtained only because irrelevant features were removed - see figure 1

Flags classification problem have 193 vectors, 28 attributes, and each vector may be assigned to one of 8 classes. Feature weighting for this data set was very efficient (see tab. 2). For 1NN CV3 with Manhattan metric accuracy in test was $62.04 \%$ and without weighting $50.91 \%$. For 3NN with weighting $61.51 \%$ and $48.07 \%$ without. The best non-weighted $\mathrm{kNN}$ have accuracy $52.56 \%$ and this is nearly $10 \%$ less than for the best plain $\mathrm{kNN}$.

Glass data contains 214 vectors, each vector has 9 attributes and may be classified to one of the 6 classes. For this benchmark weighting looks very important, and the improvement may be really large (see tab. 3). The best results were obtained for 1 NN CV3 with Manhattan metric giving $80.81 \%$ accuracy, and the plain version finished with just $73.50 \%$. For 3 neighbors (3NN CV3 Manh) weighted version has 78.04\%, and non-weighted $72.06 \%$.

The number of $\Delta$ changes change from 25 to 40 for glass data set and from 25 to 50 for flags data set (it is around 1 minute on Pentium 4).

For the following data sets results of feature weighting were not significant (around 0.3-2\%): australian credit, appendicitis, cleveland heart, wine.

\section{Conclusions}

Discrete quasi-gradient weighting algorithm presented in this paper is really efficient and results have good accuracy on several tests. Sometimes the improvement was around $10 \%$ of accuracy. This algorithm may be used as feature extraction tool. The starting feature set may be full or empty, and because of that it may be useful for huge dimensional data sets. Also alternative weighting sets may be obtained, what may be very important in further analysis. Using this feature weighting algorithm the $\mathrm{kNN}$ model was placed at the top of ranking of the best methods for presented data sets. Sometimes the

\footnotetext{
${ }^{3}$ Canberra metric: $\operatorname{canb}(\mathbf{x}, \mathbf{y})=\sum_{i=1}^{d}\left|x_{i}-y_{i}\right| /\left|x_{i}+y_{i}\right|$.
} 


\begin{tabular}{|c|c|}
\hline Model & test train \\
\hline 3NN CV3 Camberra & 98.5699 .37 \\
\hline 3NN CV2 Manh & 98.3699 .28 \\
\hline 1NN CV2 Manh & 98.26100 .00 \\
\hline $3 \mathrm{NN}$ CV2 & 98.2899 .24 \\
\hline $1 \mathrm{NN} \mathrm{CV} 2$ & 98.26100 .00 \\
\hline $2 \mathrm{NN} \mathrm{CV} 2$ & 98.3699 .28 \\
\hline 3NN Camberra & 96.3098 .49 \\
\hline 3NN Manh & 94.4096 .58 \\
\hline 1NN Manh & 93.76100 .00 \\
\hline $1 \mathrm{NN}$ & 93.14100 .00 \\
\hline default & $92.71-$ \\
\hline C-MLP2LN+ASA 11 & 99.3699 .90 \\
\hline CART [12] & 99.3699 .80 \\
\hline PVM 12 . & 99.3699 .80 \\
\hline SSV 13 & 99.3699 .76 \\
\hline IncNet 14 & 99.2499 .68 \\
\hline MLP a,b opt. 11 & 99.3699 .90 \\
\hline MLP2LN 11 & 99.0099 .70 \\
\hline Cascade correlation 15 & 98.50100 .00 \\
\hline $\mathrm{BP}+$ genetic 15 & 98.4099 .40 \\
\hline Quickprop [15] & 98.3099 .60 \\
\hline kNN+weights [10] & 98.2298 .89 \\
\hline RProp 15] & 98.0099 .60 \\
\hline kNN+weights [10] & $97.9698 .89 \ldots$ \\
\hline
\end{tabular}
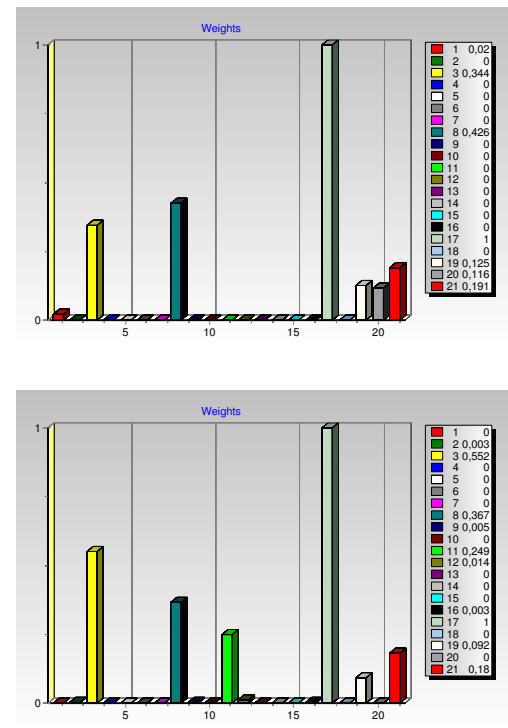

Fig. 1. Feature weights for thyroid data set.

Table 1. Accuracy for thyroid data set.

\begin{tabular}{ll} 
Model & test train \\
\hline 1NN CV3 Manh & 62.04100 .0 \\
3NN CV3 Manh & 61.5176 .07 \\
7NN CV3 Manh & 58.3068 .28 \\
27NN CV3 Manh 56.1959 .78 \\
\hline 27NN Manh & 52.5656 .53 \\
7NN Manh & 52.0363 .57 \\
1NN Manh & 50.91100 .00 \\
17NN Manh & 50.2557 .97 \\
3NN Manh & 48.0772 .53 \\
27NN & 46.7651 .75 \\
1NN & $48,52100,00$ \\
\hline default & $31.09-$ \\
\hline CART [16] & $62.40-$ \\
IB1 [16] & $56.60-$ \\
C 4.5 [16] & $56.20-$ \\
GIBL [] & $55.11-$ \\
IBL [7] & $48.29-\ldots$ \\
\hline
\end{tabular}

Table 2. Accuracy for flags data set.

\begin{tabular}{ll} 
Model & test train \\
\hline 1NN CV3 Manh & 80.81100 .00 \\
3NN CV3 Manh & 78.0487 .64 \\
3NN CV3 Mink-0.7 77.9788 .78 \\
3NN CV2 Manh & 77.4987 .53 \\
3NN CV2 & 75.0085 .11 \\
\hline 1NN Manh & 73.50100 .00 \\
3NN Manh & 72.0684 .98 \\
3NN & 70.8583 .63 \\
1NN & 70.45100 .00 \\
\hline default & $35.51-$ \\
\hline GIBL [7] & $78.55-$ \\
Bayes [16] & $71.80-$ \\
CART & $71.40-$ \\
IB1 [16] & $71.10-$ \\
ID3 [16] & $69.10-$ \\
IBL [] & $70.52-$ \\
\hline
\end{tabular}

Table 3. Accuracy for glass data set. 
accuracy on testing and training parts were very similar, which means that solution found are close to optimum for a given model. It is important that algorithm may be used not only for $\mathrm{kNN}$, but for several machine learning models, including artificial neural networks.

\section{References}

1. C. M. Bishop. Neural Networks for Pattern Recognition. Oxford University Press, 1995.

2. R. O. Duda, P. E. Hart, and D. G. Stork. Patter Classification and Scene Analysis. Wiley, 2nd ed. edition, 1997.

3. M. Dash and H. Liu. Feature selection for classification. Intelligent Data Analysis, 1(3), 1997.

4. M. Fernández and C. Hernández. How to select the inputs for a multilayer feedforward by using the training set. In 5th International Work Conference on Artificial an Natural Neural Networks, pages 477-486, Alicante, Spain, 1999.

5. H. Almuallim and T. G. Dietterich. Efficient algorithms for identifying relevant features. In Proceedings of the Ninth Canadian Conference on Artificial Intelligence, pages 38-45, Vancouver, 1992. Morgan Kaufmann.

6. D. R. Wilson and T. R. Martinez. Instance-based learning with genetically derived attribute weights. In International Conference on Artificial Intelligence, Expert Systems and Neural Networks, pages 11-14, 1996.

7. D. R. Wilson. Advances in Instance-Based Learning Algorithms. PhD thesis, Department of Computer Science Brigham Young University, 1997.

8. T. M. Cover and P. E. Hart. Nearest neighbor pattern classification. Institute of Electrical and Electronics Engineers Transactions on Information Theory, 13(1):21-27, jan 1967.

9. C. J. Merz and P. M. Murphy. UCI repository of machine learning databases, 1998. http://www.ics.uci.edu/ mlearn/MLRepository.html.

10. W. Duch and K. Grudziński. Search and global minimization in similaritybased methods. In International Joint Conference on Neural Networks, page 742, Washington, 1999.

11. W. Duch, R. Adamczak, and K. Grabczewski. Extraction of logical rules from backpropagation networks. Neural Processing Letters, 7:1-9, 1998.

12. S.M. Weiss and I. Kapouleas. An empirical comparison of pattern recognition, neural nets and machine learning classification methods. In J.W. Shavlik and T.G. Dietterich, editors, Readings in Machine Learning. Morgan Kauffman, 1990.

13. K. Grabczewski and Włodzisław Duch. The separability of split value criterion. In L. Rutkowski and R. Tadeusiewicz, editors, Neural Networks and Soft Computing, pages 202-208, Zakopane, Poland, June 2000.

14. N. Jankowski. Ontogenic neural networks and their applications to classification of medical data. PhD thesis, Department of Computer Methods, Nicholas Copernicus University, Toruń, Poland, 1999.

15. W. Schiffman, M. Joost, and R. Werner. Comparison of optimized backpropagation algorithms. In Proceedings of ESANN'93, pages 97-104, Brussels, 1993.

16. Frederick Zarndt. A comprehensive case study: An examination of machine learning and connectionist algorithms. Master's thesis, Department of Computer Science Brigham Young University, 1995. 